\title{
Water and solute absorption from a new hypotonic oral rehydration solution: evaluation in human and animal perfusion models
}

\author{
J B Hunt, A V Thillainayagam, A F M Salim, S Carnaby, E J Elliott, M J G Farthing
}

\begin{abstract}
Controversy continues regarding the optimal composition of glucose electrolyte oral rehydration solutions for the treatment of acute diarrhoea. Four perfusion models (normal human jejunum, normal rat small intestine, cholera toxin treated secreting rat small intestine and rotavirus infected rat small intestine) have been developed and used to compare the efficacy of a hypotonic oral rehydration solution with standard United Kingdom British National formulary and developing world oral rehydration solutions (WHO). Despite obvious physiological and pathophysiological differences between these models there was general congruence in the water and solute absorption profiles of the different oral rehydration solutions. Hypotonic oral rehydration solution promoted significantly greater water absorption than other oral rehydration solutions in all rat models $(\mathbf{p}<\mathbf{0 . 0 0 1})$ but apparently increased water absorption failed to achieve significance in human jejunum. British National Formularyoral rehydration solution was unable to reverse net water secretion in both rotavirus and cholera toxin models. Net sodium absorption from hypotonic and WHO-oral rehydration solutions was signficantly greater than from the low sodium British National Formulary-oral rehydration solutions $(p<0.001)$ except in the rotavirus model when absorption was similar to hypotonic-oral rehydration solutions. These findings show that there is agreement in the apparent efficacy of oral rehydration solutions in these animal and human perfusion models, and that improved water absorption with adequate sodium absorption may be achieved by reducing oral rehydration solution osmolality.
\end{abstract}

(Gut 1992; 33: 1652-1659)

Departments of

Gastroenterology and

Paediatric

Gastroenterology, St

Bartholomew's Hospital,

West Smithfield, London

J B Hunt

A V Thillainayagam

A F M Salim

S Carnaby

E J Elliott

M J G Farthing

Correspondence to:

Professor M J G Farthing,

Department of

Gastroenterology,

St Bartholomew's Hospital,

West Smithfield, London

EClA 7BE.

Accepted for publication

19. May 1992
Establishment of the efficacy of glucose electrolyte oral rehydration solutions in the late $1960 \mathrm{~s}^{1-3}$ and their further development ${ }^{+}$has been one of the major health care advances of the last half century. While the currently available formulations are highly effective in rehydrating patients with acute diarrhoea of diverse aetiologies,,$^{5 h}$ an optimal formulation for both correction of dehydration and maintenance of rehydration has not been determined. Furthermore, neither the World Health Organisation recommended oral rehydration solution nor any of the commercially available oral rehydration solutions reduce stool volume. This desirable effect has been reported by some ${ }^{7 . x}$ but not all workers" who have studied cereal based solutions. Such solutions are by their nature hypotonic whereas most currently available oral rehydration solutions are mildly or moderately hypertonic.

The ideal method for assessing new oral rehydration solutions is by clinical trial. "101" Such studies are highly demanding and thus the number of solutions which can be tested is limited. Several authors have used animal perfusion models of either healthy" ${ }^{14}$ or 'discased' intestine $^{14}$ to assess the ability of oral rehydration solutions to promote water and sodium absorption, while others have evaluated oral rehydration solutions by human jejunal perfusion. ${ }^{1617}$ The comparability between these different model perfusion systems is unknown as is their relevance to the behaviour of oral rehydration solutions in acute diarrhoea. Despite their inherent limitations such studies are the only intermediate form of assessment of oral rehydration solutions that is available.

We have, therefore, compared net water and solute absorption from three oral rehydration solutions in four perfusion systems: (i) adult rat entire small intestine, (ii) cholera toxin treated adult rat entire small intestine, (iii) rotavirus infected neonatal rat small intestine and (iv) human triple lumen jejunal perfusion. The oral rehydration solutions tested were WHO oral rehydration solutions, the oral rehydration solutions recommended by the British National Formulary, British National Formulary-oral rehydration solution, which is the most widely used in the United Kingdom and a new, experimental hypotonic oral rehydration solution.

\section{Methods}

\section{SOLUTION DESIGN}

The composition of the oral rehydration solutions is shown in Table I. The sodium concentration of WHO oral rehydration solution is relatively high $(90 \mathrm{mmol} / \mathrm{l})$. While this approximates to the minimum concentration necessary to ensure sodium absorption from isotonic solutions in a short segment of healthy human jejunum, ${ }^{1 x}$ the use of the WHO oral rehydration solutions clinically has been associated occasionally with hypernatraemia." The WHO has therefore recommended that once rehydration has been achieved, free water is administered with WHO oral rehydration solutions during the maintenance period." This complicates oral rehydration therapy but when correctly followed it effectively results in the presentation to the small bowel of a hypotonic solution with a lower 
TABLE I Composition of solutions perfused

\begin{tabular}{lccccc}
\hline Solute (mmol/l) & HYPO-ORS & BNF-ORS & WHO-ORS & PES & NaCl \\
\hline Sodium & 60 & 35 & 90 & $1+0$ & $1+5$ \\
Glucose & 90 & 200 & 111 & - & - \\
Potassium & 20 & 20 & 20 & 4 & - \\
Bicarbonate & - & 18 & - & +0 & - \\
Citrate & 20 & - & 80 & - & - \\
$\begin{array}{l}\text { Chloride } \\
\text { Calculated osmolality } \\
\quad(\text { mOsmol/kg) }\end{array}$ & 60 & 37 & 331 & 288 & 290 \\
\hline
\end{tabular}

All solutions contained $2 \cdot 5-5 \mathrm{~g} / \mathrm{l}$ polyethylene glycol (PEG) +000 and $2-5 \mu \mathrm{Ci} / \mathrm{l}\left[{ }^{+} \mathrm{C}\right]-\mathrm{PEG}$.

HYPO: hypotonic; BNF: British National Formulary; WHO: World Health Organisation; PES: plasma electrolyte solution; $\mathrm{NaCl}$, isotonic saline.

sodium, glucose, and potassium concentration. It would be simpler to use a single solution without alteration throughout the rehydration and the maintenance periods. If this were possible compliance might be improved.

The sodium concentration $(35 \mathrm{mmol} / \mathrm{l})$ in British National Formulary oral rehydration solution is far below the concentration necessary to prevent passive sodium entry into the jejunum in healthy adults. ${ }^{20}$ Therefore sodium entry into the jejunum must be expected with this solution. This, in addition to sodium secretion induced by the diarrhoeal illness will partially nullify the benefits of solute linked sodium absorption produced by the glucose in oral rehydration solutions. The glucose concentration (200 $\mathrm{mmol} / \mathrm{l}$ ) of British National Formulary oral rehydration solution is almost certainly too high..$^{.1}$ It is more than twice the value required to maximally stimulate sodium absorption in jejunal perfusion studies in healthy volunteers ${ }^{22}$ and solutions with this glucose concentration limit sodium and water absorption when compared with solutions with lower glucose concentrations. $^{2223}$ When oral rehydration solutions with similar glucose concentrations have been used in adults ${ }^{24}$ or children ${ }^{24}$ with acute diarrhoea, reducing substances (glucose) have been detected in the stools of some of the patients. It is suggested that glucose may have promoted osmotic diarrhoea and exacerbated water loss. ${ }^{21}$ This would detract from the efficacy of the oral rehydration solutions and decrease net solute and water absorption.

Both WHO oral rehydration solution and British National Formulary oral rehydration solution are hypertonic with respect to human plasma (331 and $310 \mathrm{mOsmol} / \mathrm{kg}$, respectively). As the proximal small bowel is unable to support an osmotic gradient ${ }^{26}$ these solutions may promote secretion of fluid. We have designed a new hypotonic solution with a sodium concentration of $60 \mathrm{mmol} / \mathrm{l}$ and calculated osmolality of 240 $\mathrm{mOsmol} / \mathrm{kg}$. This sodium concentration was chosen as it has been shown to be effective in acute childhoood diarrhoea ${ }^{27}$ and is the concentration of sodium which has been advocated as optimal for oral rehydration therapy. ${ }^{28}$ The glucose concentration $(90 \mathrm{mmol} / \mathrm{l})$ is less than that of WHO oral rehydration solution (111 $\mathrm{mmol} / \mathrm{l}$ ) and British National Formulary oral rehydration solution $(200 \mathrm{mmol} / \mathrm{l})$. This ensured the hypotonicity of hypotonic oral rehydration solution which we postulated would increase water absorption and possibly ultimately decrease stool volume. We also included citrate in place of bicarbonate (as now recommended by WHO) as it is both more stable during storage than bicarbonate and may be used as a disodium salt, thus allowing further reduction in osmolality. Perfusion studies in man have shown citrate to enhance absorption ${ }^{34}$ and citrate containing oral rehydration solutions have been successfully used in clinical studies. : $^{\prime \prime}$ All solutions contained $2 \cdot 5-5 \cdot 0 \mathrm{~g} / 1$ polyethylene glycol 4000 and $2-5 \cdot 0 \mu \mathrm{Ci} / 1\left[{ }^{1+} \mathrm{C}\right]$ polyethylene glycol.

We have examined net water and electrolyte absorption from this candidate solution and compared it with that seen with British National Formulary-oral rehydration solution and the WHO oral rehydration solution in four in vivo perfusion models. We also perfused a glucose free isotonic sodium chloride solution or a plasma electrolyte solution to establish basal transport of water and electrolytes in each model.

\section{INTESTINAL PERFUSION \\ (i) Perfusion of adult rat entire small intestine}

As previously described" male adult Wistar rats (weight 200-220 g) were subjected to laparotomy under pentobartibone anaesthesia. Both the duodenum and distal ileum were cannulated and the entire small intestine perfused at $30 \mathrm{ml} /$ h. After a 60 minute equilibration period, three 10 minute collections were made. At the end of the experiment the animals were killed by intracardiac injection of pentobartitone. The perfused segment of intestine was then removed, rinsed and dried in an oven at $90^{\circ} \mathrm{C}$ for 18 hours. polyethylene glycol recovery in these experiments was $100 \cdot 3(3) \%$.

TABLE IIA Solute concentrations (Median Iinterquartile range $/ \mathrm{mmol} / \mathrm{l}$ ) at entry to the test segment in human jejunum

\begin{tabular}{llll}
\hline & HYPO-ORS & BNF-ORS & WHO-ORS \\
\hline Sodium & $75 \cdot 1$ & $52 \cdot 8$ & $96 \cdot 2$ \\
& $(71 \cdot 1-80 \cdot 7)$ & $(48 \cdot 6-55 \cdot 3)$ & $(94 \cdot 7-99 \cdot 1)$ \\
Potassium & $18 \cdot 8$ & $15 \cdot 9$ & $16 \cdot 7$ \\
& $(18 \cdot 5-19 \cdot 3)$ & $(15 \cdot 4-16 \cdot 6)$ & $(15 \cdot 9-16 \cdot 8)$ \\
Bicarbonate & ND & $10 \cdot 4$ & $16 \cdot 1$ \\
& & $(9 \cdot 7-15 \cdot 1)$ & $(15 \cdot 6-17 \cdot 1)$ \\
Citrate & ND & ND & NI) \\
Chloride & 74 & 51 & 84 \\
& $(68-75)$ & $(50-53)$ & $(81-86)$ \\
Glucose & 65 & 159 & 87 \\
& $(63-74)$ & $(155-166)$ & $(71-89)$ \\
Measured osmolality & 245 & 290 & 283 \\
(mOsmol/kg) & $(238-256)$ & $(283-294)$ & $(274-287)$ \\
\hline
\end{tabular}

ND: not determined.

TABLE IIB Calculated solute concentrations (Median [interquartile range] mmol/l) at midpoint of the test segment in normal rat small intestine

\begin{tabular}{|c|c|c|c|}
\hline & HYPO-ORS & BNF-ORS & WHO-(ORSS \\
\hline Sodium & $\begin{array}{l}84 \cdot 6 \\
(83 \cdot 2-86 \cdot 3)\end{array}$ & $\begin{array}{l}41 \cdot 2 \\
(42 \cdot 2-42 \cdot 0)\end{array}$ & $\begin{array}{l}93.5 \\
(90 \cdot 8-99 \cdot 7)\end{array}$ \\
\hline Potassium & $\begin{array}{l}17 \cdot 0 \\
(16 \cdot 5-17 \cdot 8)\end{array}$ & $\begin{array}{l}15 \cdot 6 \\
(15 \cdot 1-16 \cdot 5)\end{array}$ & $\begin{array}{l}16 \cdot 4 \\
(15 \cdot 5-17 \cdot 9)\end{array}$ \\
\hline Bicarbonate & ND & $\begin{array}{l}11 \cdot 7 \\
(11 \cdot 2-12 \cdot 0)\end{array}$ & $\begin{array}{l}25 \cdot 4 \\
(20 \cdot 1-28 \cdot 0)\end{array}$ \\
\hline Citra & ND & NI) & NI) \\
\hline Chlo & $\begin{array}{l}71 \cdot 5 \\
(69 \cdot 2-73 \cdot 6)\end{array}$ & $\begin{array}{l}41 \cdot 6 \\
(40 \cdot 7-42 \cdot 0)\end{array}$ & $\begin{array}{l}86 \cdot 8 \\
(86 \cdot 7-87 \cdot 7)\end{array}$ \\
\hline Glucose & $\begin{array}{l}53 \cdot 1 \\
(45 \cdot 9-55 \cdot 2)\end{array}$ & $\begin{array}{l}192 \cdot 0 \\
(187 \cdot 1-192 \cdot 0)\end{array}$ & $\begin{array}{l}94 \cdot 6 \\
(92 \cdot 2-95 \cdot 9)\end{array}$ \\
\hline $\begin{array}{l}\text { Osmolality } \\
(\mathrm{mOsmol} / \mathrm{kg})\end{array}$ & $\begin{array}{l}259 \cdot 7 \\
(256 \cdot 9-261 \cdot 0)\end{array}$ & NI) & NI) \\
\hline
\end{tabular}

ND: not determined. 
(ii) Cholera toxin treated adult rat entire small intestine

An identical operative procedure was used to that outlined in (i). As described previously $75 \mu \mathrm{g}$ cholera toxin (Sigma Chemical Company Ltd, Poole, Dorset) was used to induce a secretory state. ${ }^{15}$ After two hours, the bowel was cleansed

TABLE IIC Calculated solute concentrations (median [interquartile range] mmol/l) at midpoint of the test segment in chorera toxin treated rat small intestine

\begin{tabular}{llll}
\hline & HYPO-ORS & BNF-ORS & WHO-ORS \\
\hline Sodium & $75 \cdot 4$ & $41 \cdot 4$ & $101 \cdot 5$ \\
& $(74 \cdot 2-76 \cdot 3)$ & $(37 \cdot 8-43 \cdot 4)$ & $(100 \cdot 1-102 \cdot 2)$ \\
Potassium & $18 \cdot 8$ & $15 \cdot 0$ & $18 \cdot 1$ \\
& $(17 \cdot 9-18 \cdot 7)$ & $(14 \cdot 1-15 \cdot 4)$ & $(16 \cdot 9-18 \cdot 4)$ \\
Bicarbonate & $\mathrm{ND}$ & $13 \cdot 8$ & $30 \cdot 7$ \\
& & $(13 \cdot 6-16 \cdot 1)$ & $(29 \cdot 0-31 \cdot 3)$ \\
Citrate & $\mathrm{ND}$ & $\mathrm{ND}$ & $\mathrm{ND}$ \\
Chloride & $67 \cdot 4$ & $37 \cdot 9$ & $87 \cdot 1$ \\
& $(65 \cdot 7-68 \cdot 6)$ & $(32 \cdot 8-42 \cdot 2)$ & $(86 \cdot 2-85 \cdot 9)$ \\
Glucose & $67 \cdot 7$ & $178 \cdot 3$ & $81 \cdot 6$ \\
& $(75 \cdot 3-79 \cdot 0)$ & $(173 \cdot 8-181 \cdot 0)$ & $(78 \cdot 2-84 \cdot 9)$ \\
Osmolality & $255 \cdot 6$ & $\mathrm{ND}$ & $\mathrm{ND}$ \\
$\quad$ (mOsmol/kg) & $(254 \cdot 4-256 \cdot 6)$ & & \\
\end{tabular}

ND: not determined.

TABLE IID Calculated solute concentrations (median [interquartile range] mmol/l) at midpoint of the test segment in rotavirus infected rat small intestine

\begin{tabular}{llll}
\hline & HYPO-ORS & BNF-ORS & WHO-ORS \\
\hline Sodium & $60 \cdot 4$ & $36 \cdot 5$ & $88 \cdot 7$ \\
& $(59 \cdot 6-61 \cdot 2)$ & $(36 \cdot 1-36 \cdot 6)$ & $(88 \cdot 4-88 \cdot 8)$ \\
Potassium & $18 \cdot 2$ & $16 \cdot 5$ & $18 \cdot 6$ \\
& $(18 \cdot 2-18 \cdot 8)$ & $(15 \cdot 8-16 \cdot 6)$ & $(18 \cdot 4-18 \cdot 6)$ \\
Citrate & $\mathrm{ND}$ & $\mathrm{ND}$ & $\mathrm{ND}$ \\
Chloride & $6 \cdot 1$ & $37 \cdot 1$ & $80 \cdot 8$ \\
& $(61-61 \cdot 4)$ & $(36 \cdot 9-37 \cdot 3)$ & $(80 \cdot 4-80 \cdot 9)$ \\
Glucose & 90 & $177 \cdot 7$ & $100 \cdot 6$ \\
& $(89 \cdot 7-92 \cdot 2)$ & $(177 \cdot 7-178 \cdot 0)$ & $(99 \cdot 5-102 \cdot 2)$ \\
Osmolality & $\mathrm{ND}$ & $\mathrm{ND}$ & $\mathrm{ND}$ \\
\multicolumn{1}{c}{ (m)smol/kg) } & & & \\
& & & \\
\hline
\end{tabular}

ND: not determined.

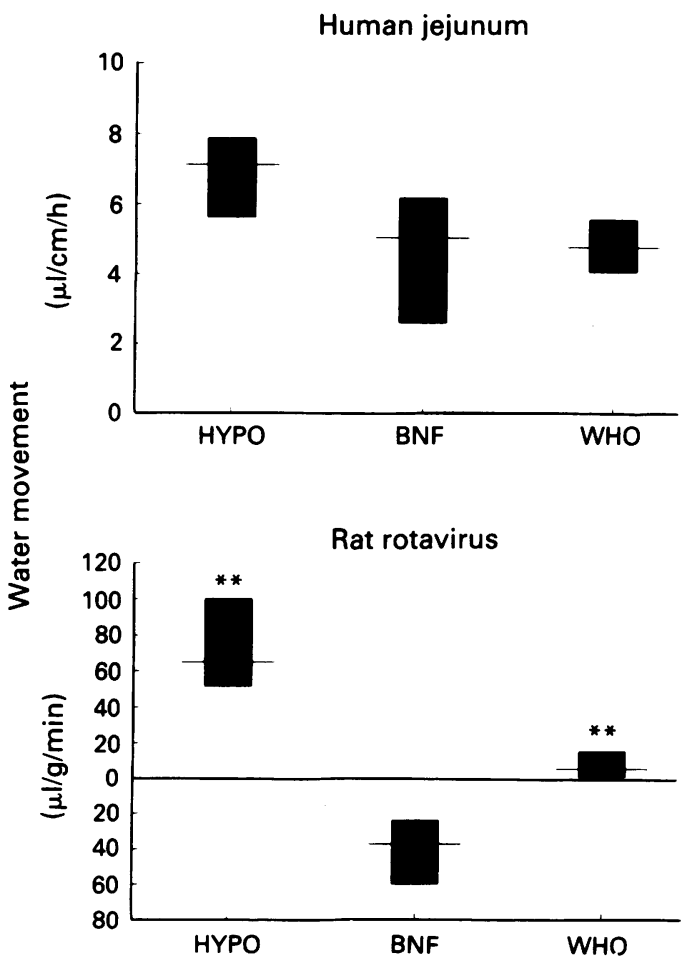

with $10 \mathrm{ml}$ test solution injected slowly to remove accumulated mucus. Perfusion was then performed as described in (i). Polyethylene glycol recovery was $101 \cdot 2(1 \cdot 9) \%$.

\section{(iii) Rotavirus infected neonatal rat entire small} intestine

Eight day old suckling Wistar rats were gavaged with group B rotavirus (obtained initially from Dr S L Vonderfecht, John Hopkins School of Medicine, Baltimore, Md, USA) in $0.25 \mathrm{ml}$ water. The animals were returned to their dams and 48 hours later anaesthetised with pentobarbitone $30 \mathrm{mg} / \mathrm{kg}$ intraperitoneally. We have shown previously that both jejunal mucosal damage and a secretory state were consistently established at this time. ${ }^{31}$ Rats then underwent laparotomy and the entire small intestine prepared and perfused as in (i). The perfusion rate was reduced to $15 \mathrm{ml} /$ hour because of the

TABLE IIE Calculated solute concentrations (median [interquartile range] mmol/l) at midpoint of the test segment in human jejunum

\begin{tabular}{|c|c|c|c|}
\hline & HYPO-ORS & $B N F-O R S$ & WHO-ORS \\
\hline dium & $\begin{array}{l}81 \cdot 4 \\
(76 \cdot 3-89 \cdot 0)\end{array}$ & $\begin{array}{l}58 \cdot 6 \\
(54 \cdot 1-62 \cdot 0)\end{array}$ & $\begin{array}{l}100 \cdot 0 \\
(97 \cdot 6-104 \cdot 6)\end{array}$ \\
\hline Potassium & $\begin{array}{l}19 \cdot 4 \\
(18 \cdot 5-19 \cdot 2)\end{array}$ & $\begin{array}{l}14 \cdot 0 \\
(13 \cdot 3-15 \cdot 3)\end{array}$ & $\begin{array}{l}15 \cdot 5 \\
(15 \cdot 2-16 \cdot 1)\end{array}$ \\
\hline Bicarbonate & ND & $\begin{array}{l}14 \cdot 5 \\
(12 \cdot 7-16 \cdot 2)\end{array}$ & $\begin{array}{l}15 \cdot 2 \\
(15 \cdot 1-16 \cdot 4)\end{array}$ \\
\hline $\begin{array}{l}\text { Citrate } \\
\text { Chloride }\end{array}$ & $\begin{array}{l}\text { ND } \\
77 \cdot 9 \\
(73 \cdot 0-82 \cdot 6)\end{array}$ & $\begin{array}{l}\text { ND } \\
54 \cdot 0 \\
(52 \cdot 9-57 \cdot 7)\end{array}$ & $\begin{array}{l}\text { ND } \\
86 \cdot 8 \\
(83 \cdot 5-89 \cdot 4)\end{array}$ \\
\hline Glucose & $\begin{array}{l}57 \cdot 0 \\
(51 \cdot 0-71 \cdot 5)\end{array}$ & $\begin{array}{l}140 \cdot 2 \\
(129 \cdot 1-142 \cdot 3)\end{array}$ & $\begin{array}{l}65 \cdot 6 \\
(56 \cdot 8-79 \cdot 6)\end{array}$ \\
\hline $\begin{array}{l}\text { Osmolality } \\
(\mathrm{mOsmol} / \mathrm{kg})\end{array}$ & $\begin{array}{l}250 \cdot 4 \\
(243 \cdot 9-262 \cdot 4)\end{array}$ & $\begin{array}{l}289 \cdot 5 \\
(268 \cdot 7-292 \cdot 3)\end{array}$ & $\begin{array}{l}278 \cdot 2 \\
(272 \cdot 2-287 \cdot 7)\end{array}$ \\
\hline
\end{tabular}

ND: not determined.
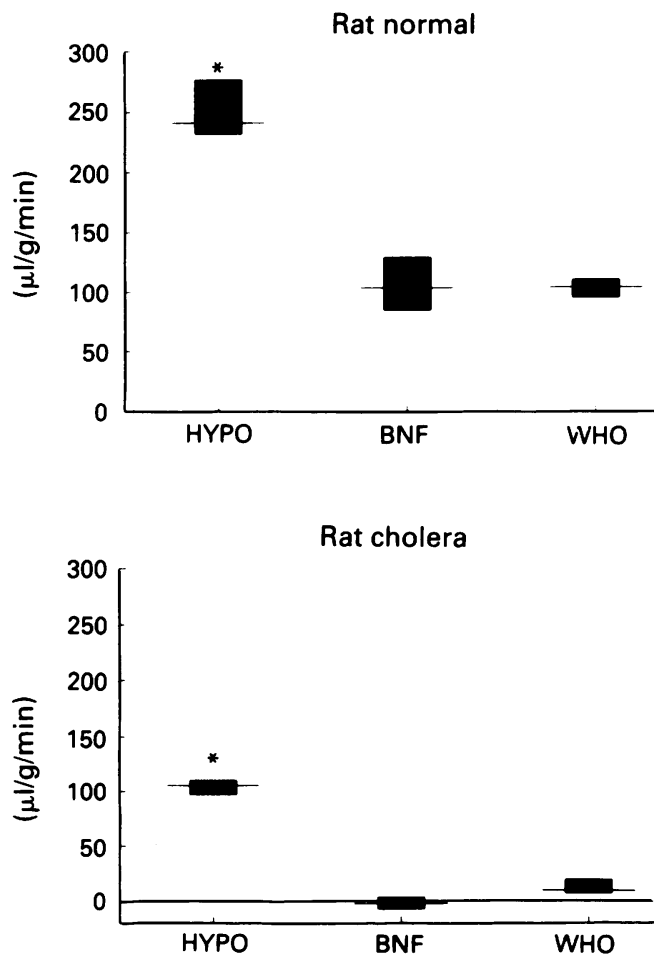

Solution

Figure 1: Net water movement during perfusion of hypotonic (HYPO), British National Formulary (BNF) and World Health Organisation (WHO) oral rehydration solutions for four perfusion models (normal human jejunum, normal and cholera toxin treated rat small intestine and rotavirus infected neonatal rat small intestine $) .{ }^{\star} p<0.001 ;{ }^{\star} p<0.01$. 
small luminal capacity of the intestine. Polyethylene glycol recovery was $97 \cdot 0(0 \cdot 4) \%$.

\section{(iv) Human triple lumen jejunal perfusion}

Triple lumen jejunal perfusion were performed as previously described ${ }^{16}$ in healthy volunteers (ages 18-31 years, 11 men, 14 women) who gave written informed consent. This study was approved by the Research Ethics Committee of the City and Hackney Health District. A $10 \mathrm{~cm}$ mixing and $30 \mathrm{~cm}$ test segment were used. Solutions under test were perfused at $15 \mathrm{ml} /$ minute by a Watson-Marlow flow inducer which was calibrated before each study. After a 30 minute equilibration period, three sequential 10 minute collections were made. Collection from the proximal port was by hand held syringe, aspirated at $1.5 \mathrm{ml} /$ minute and from the distal port by passive siphonage. As a result of preliminary studies which documented a transit time of $4 \cdot 1(0 \cdot 6)$ minutes, collection from the two ports was staggered by 10 minutes to allow for transit of fluid. The solutions were perfused in a predetermined, but randomised order.

In keeping with other workers ${ }^{32}$ an arbitrary limit of $>70 \%$ of expected recovery from the proximal port and $>30 \%$ of remaining marker recovery from the distal port was set for the human triple lumen perfusions.

The composition of all oral rehydration solutions changed with transit through the mixing segment of human jejunum (Table IIa) and the perfusion test segments (Tables IIb-e). The sodium and chloride concentrations in all solutions increased but the relative initial difference between oral rehydration solutions were maintained at the midpoint of the perfusion segment. The potassium and bicarbonate concentrations of all solutions decreased slightly. Glucose concentration also decreased in all solutions but by similar proportions so the relative differences in glucose concentration were preserved. These alterations in solute concentration resulted in changes in the osmolality of all solutions towards isotonicity.

\section{ANALYSIS OF SPECIMENS}

Specimens were analysed on the day of perfusion. Bicarbonate was determined immediately using a Corning $\mathrm{CO}_{2}$ meter. Sodium and potassium analysis was by flame photometry (Instrumentation Laboratory 943). Chloride was analysed using a Corning chloride analyser 925 and osmolality on a Wescor 5500 vapour pressure osmometer. Polyethylene glycol concentrations were measured in triplicate on an Ultrabeta 1210 scintillation counter (LKB Wallac) using $0.5 \mathrm{ml}$ aliquots of sample in $4.5 \mathrm{ml}$ of laboratory prepared scintillation fluid. Samples from human perfusion were analysed using a quench correction program to negate any effects caused by bile contamination of the perfusion fluid. The quench correction was constructed using bile obtained at a routine cholecystectomy and $\left[{ }^{1+} \mathrm{C}\right]$ seed pellets of known activity (LKB Wallac).

\section{CALCULATIONS AND STATISTICAL ANALYSIS}

Net water and solute movement were calculated using standard formulae. ${ }^{212}$ Results were analysed statistically using the Kruskal-Wallis
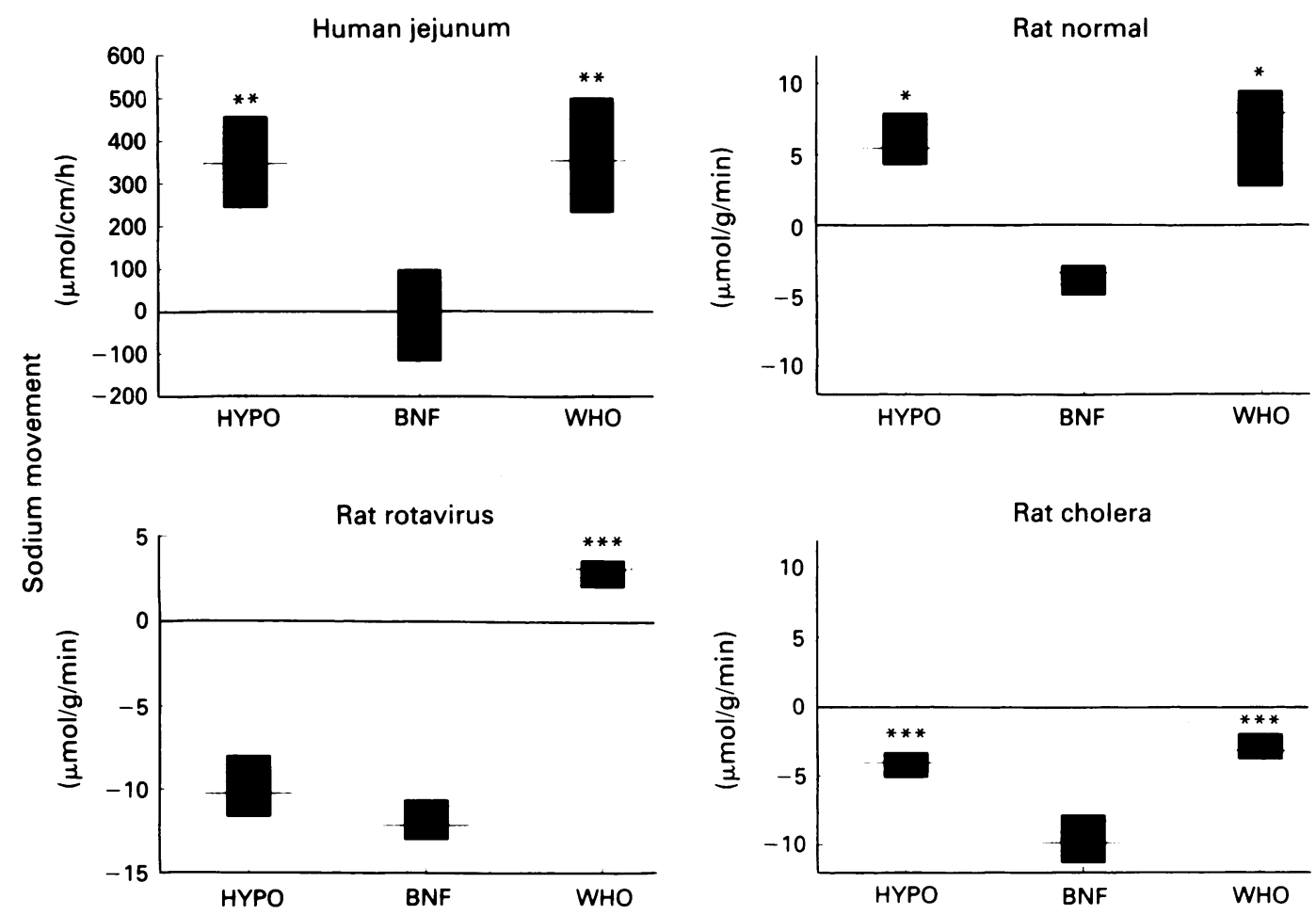

Solution

Figure 2: Net sodium movement during perfusion of hypotonic ( $H Y P O)$, British National Formulary (BNF) and World Health Organisation (WHO) oral rehydration solutions in four perfusion models (normal human jejunum, normal and cholera toxin treated rat small intestine and rotavirus infected neonatal rat small intestine. ${ }^{\star} p<0 \cdot 001 ;{ }^{\star \star} p<0 \cdot 01 ;{ }^{\star}{ }^{\circ} p<0 \cdot 05$. 
test for non-parametric data ${ }^{33}$ and values for the various parameters measured are expressed as medians and interquartile ranges. Correction was made for multiple comparisons. All treatments have been compared within each model. Positive values reflect absorption from the lumen.

\section{Results}

Plasma electrolyte solution promoted net water, sodium and chloride absorption in both normal human jejunum (water $+3.6(2.0$ to 4.0$) \mathrm{ml} /$ $\mathrm{cm} / \mathrm{h}$; sodium, $+360(322$ to 400$) \mu \mathrm{mol} / \mathrm{cm} / \mathrm{h}$ ) and normal entire rat small intestine (water, $+3 \cdot 9(-8 \cdot 1$ to $+18 \cdot 5) \mu \mathrm{l} / \mathrm{min} / \mathrm{g}$; sodium, $+5 \cdot 3$ $(+2.9$ to +7.5$) \mu \mathrm{l} / \mathrm{min} / \mathrm{g}$. Perfusion of isotonic sodium chloride solution confirmed the presence of a secretory state in the cholera model for both water $(-47.5(-55.4$ to $-38 \cdot 3) \mu \mathrm{l} / \mathrm{min} / \mathrm{g})$ and sodium $(-3 \cdot 2(-5 \cdot 1$ to $-2 \cdot 9) \mu \mathrm{mol} / \mathrm{min} / \mathrm{g})$. In the rotavirus model water secretion occurred $(-15 \cdot 2(-1.9$ to $-41 \cdot 6) \mu \mathrm{l} / \mathrm{min} / \mathrm{g})$ with sodium

TABLE III Net solute movement (median /interquartile range] in normal rat small intestine

\begin{tabular}{llll}
\hline & HYPO-ORS & BNF-ORS & WHO-ORS \\
\hline Potassium & $5 \cdot 3$ & $7 \cdot 4^{\star}$ & $5 \cdot 0$ \\
$(\mu \mathrm{mol} / \mathrm{g} / \mathrm{min})$ & $(5 \cdot 1-5 \cdot 9)$ & $(6 \cdot 4-7 \cdot 6)$ & $(3 \cdot 6-5 \cdot 1)$ \\
Chloride & $10 \cdot 9$ & $-3 \cdot 5 \star$ & $3 \cdot 4$ \\
$(\mu \mathrm{mol} / \mathrm{g} / \mathrm{min})$ & $(9 \cdot 8-12 \cdot 8)$ & $(-5 \cdot 7--2 \cdot 2)$ & $(3 \cdot 0-5 \cdot 7)$ \\
Bicarbonate & ND & $5 \cdot 5$ & $5 \cdot 3$ \\
$(\mu \mathrm{mol} / \mathrm{g} / \mathrm{min})$ & & $(4 \cdot 7-6 \cdot 4)$ & $(4 \cdot 6-7 \cdot 1)$ \\
\hline
\end{tabular}

ND: not determined; ${ }^{\star} \mathrm{p}<0.001$ ( $v$ HYPO- and WHO-ORS) Positive values denote absorption from, negative values secretion into the lumen.

TABLE IV Net solute movement (median [interquartile range] in cholera toxin treated rat small intestine

\begin{tabular}{clll}
\hline & HYPO-ORS & BNF-ORS & WHO-ORS \\
\hline Potassium & $2 \cdot 8$ & $3 \cdot 3$ & $3 \cdot 2$ \\
$(\mu \mathrm{mol} / \mathrm{g} / \mathrm{min})$ & $(2 \cdot 6-3 \cdot 8)$ & $(3 \cdot 0-3 \cdot 5)$ & $(2 \cdot 8-3 \cdot 7)$ \\
Chloride & $0 \cdot 9$ & $-5 \cdot 9 \star$ & $0 \cdot 7$ \\
$(\mu \mathrm{mol} / \mathrm{g} / \mathrm{min})$ & $(-0 \cdot 1-2 \cdot 4)$ & $(-7 \cdot 6--4 \cdot 8)$ & $(-0 \cdot 1-1 \cdot 2)$ \\
$\begin{array}{c}\text { Bicarbonate } \\
(\mu \mathrm{mol} / \mathrm{g} / \mathrm{min})\end{array}$ & ND & 0 & $-0 \cdot 8$ \\
& & $(-0 \cdot 9-0 \cdot 7)$ & $(-1 \cdot 0-0 \cdot 01)$ \\
\hline
\end{tabular}

ND: not determined; ${ }^{\star} \mathrm{p}=0.001$ ( $v$ HYPO- and WHO-ORS) Positive values denote absorption from and negative values secretion into the lumen.

TABLE $\mathrm{V}$ Net solute movement (median /interquartile range]) in rotavirus infected rat small intestine

\begin{tabular}{llll}
\hline & HYPO-ORS & BNF-ORS & WHO-ORS \\
\hline Potassium & $1 \cdot 9$ & $-37 \cdot 1$ & $5 \cdot 8$ \\
$\begin{aligned}(\mu \mathrm{mol} / \mathrm{g} / \mathrm{min}) \\
\text { Chloride }\end{aligned}$ & $(0 \cdot 4-3 \cdot 6)$ & $(8 \cdot 7-9 \cdot 8)$ & $(5 \cdot 5-6 \cdot 0)$ \\
$\begin{array}{c}(\mu \mathrm{mol} / \mathrm{g} / \mathrm{min}) \\
\text { Bicarbonate } \\
(\mu \mathrm{mol} / \mathrm{g} / \mathrm{min})\end{array}$ & $\mathrm{ND}$ & $-3 \cdot 3^{\star}$ & $2 \cdot 5 \dagger$ \\
& & $\mathrm{ND}$ & $\mathrm{ND}$ \\
\hline
\end{tabular}

ND: not determined; ${ }^{\star} \mathrm{p}<0.001$ ( $v$ HYPO- and WHO-ORS) tp $<0.01$ ( $v$ HYPO-ORS). Positive values denote absorption from and negative values secretion into the lumen.

TABLE VI Net solute movement (median [interquartile range]) in human jejunum

\begin{tabular}{clll}
\hline & HYPO-ORS & BNF-ORS & WHO-ORS \\
\hline Potassium & $120 \cdot 3$ & $123 \cdot 4$ & $12+\cdot 6$ \\
$(\mu \mathrm{mol} / \mathrm{cm} / \mathrm{h})$ & $(104 \cdot+158 \cdot 8)$ & $(100 \cdot 7-180 \cdot 4)$ & $(96 \cdot 7-129 \cdot 1)$ \\
Chloride & $366 \cdot 0$ & $67 \cdot 0 \star$ & $27+0$ \\
$(\mu \mathrm{mol} / \mathrm{cm} / \mathrm{h})$ & $(265 \cdot 9+452 \cdot 8)$ & $(-126 \cdot 3-149 \cdot 0)$ & $(19+-399)$ \\
Bicarbonate & ND & $92 \cdot 1$ & $108 \cdot 2$ \\
$(\mu \mathrm{mol} / \mathrm{cm} / \mathrm{h})$ & & $(70 \cdot 6-125 \cdot 8)$ & $(100 \cdot 2-130 \cdot 4)$ \\
\hline
\end{tabular}

ND: not determined; ${ }^{\star}$ p $<0.01$ ( $z$, HYPO- and WHO-ORS) Positive values denote absorption from and negative values secretion into the lumen. absorption $+6 \cdot 6(+3 \cdot 5$ to $+9 \cdot 3) \mu \mathrm{mol} / \mathrm{min} / \mathrm{g})$ during perfusion of plasma electrolyte solution.

In all model systems water absorption was greatest from hypotonic-oral rehydration solution. This effect was significant in all animal systems $(\mathrm{p}<0.001)$ (Fig 1). In both the secreting models (rat cholera toxin and rat rotavirus) perfusion of British National Formulary oral rehydration solution was associated with net secretion of water. Only in the rat rotavirus model did WHO oral rehydration solution promote greater water absorption $(5 \cdot 8 \mu \mathrm{l} / \mathrm{g} / \mathrm{min})$ than British National Formulary-oral rehydration solution $(-37 \cdot 1 \mu \mathrm{l} / \mathrm{g} / \mathrm{min} ; \mathrm{p}<0.001)$.

Net sodium absorption (Fig 2) was greater from hypotonic oral rehydration solution and WHO oral rehydration solution than from British National Formulary-oral rehydration solution in rat and human normal intestine $(p<0 \cdot 001)$. The effect of the high sodium content of WHO oral rehydration solution became apparent in the secretory models where it either promoted absorption (rat rotavirus) or minimal secretion (rat cholera toxin). In the rat rotavirus model hypotonic-oral rehydration solution produced similar net sodium secretion as British National Formulary-oral rehydration solutions. In the rat cholera toxin model, perfusion of all oral rehydration solutions resulted in net sodium secretion but the effect was less marked with WHO oral rehydration solutions $(-3.2 \mu \mathrm{mol} /$ $\mathrm{g} / \mathrm{min}$ ) and hypotonic-oral rehydration solutions $(-4 \cdot 1 \mu \mathrm{mol} / \mathrm{g} / \mathrm{min})$ than British National Formulary-oral rehydration solution (-9.9 $\mu \mathrm{mol} / \mathrm{g} / \mathrm{min} ; \mathrm{p}<0.05$ ).

Differences in net potassium absorption were found only in the normal rat and rat rotavirus models, when British National Formulary-oral rehydration solution promoted greater net potassium absorption $(p<0.001)$ than either of the other two oral rehydration solutions (Tables III and V).

Only in normal human intestine was chloride absorption seen with British National Formularyoral rehydration solution. All other models showed net chloride secretion (Tables III-VI). WHO oral rehydration solution and hypotonicoral rehydration solutions promoted equivalent chloride movement in all models except rat rotavirus where chloride absorption was greater from WHO-oral rehydration solution $(\mathrm{p}<0.01)$. The bicarbonate containing solutions (British National Formulary-oral rehydration solutions and WHO oral rehydration solutions promoted similar bicarbonate absorption in human and rat intestine (Tables III-VI).

Glucose absorption was greater from British National Formulary-oral rehydration solution than either of the other solutions in both models of normal intestine and greater from WHO-oral rehydration solution in the rat rotavirus model (Fig III).

\section{Discussion}

Our findings in all model systems suggest that the use of hypotonic solutions may increase net water absorption above that seen with currently available oral rehydration solutions. This is concordant with the results of other workers in 

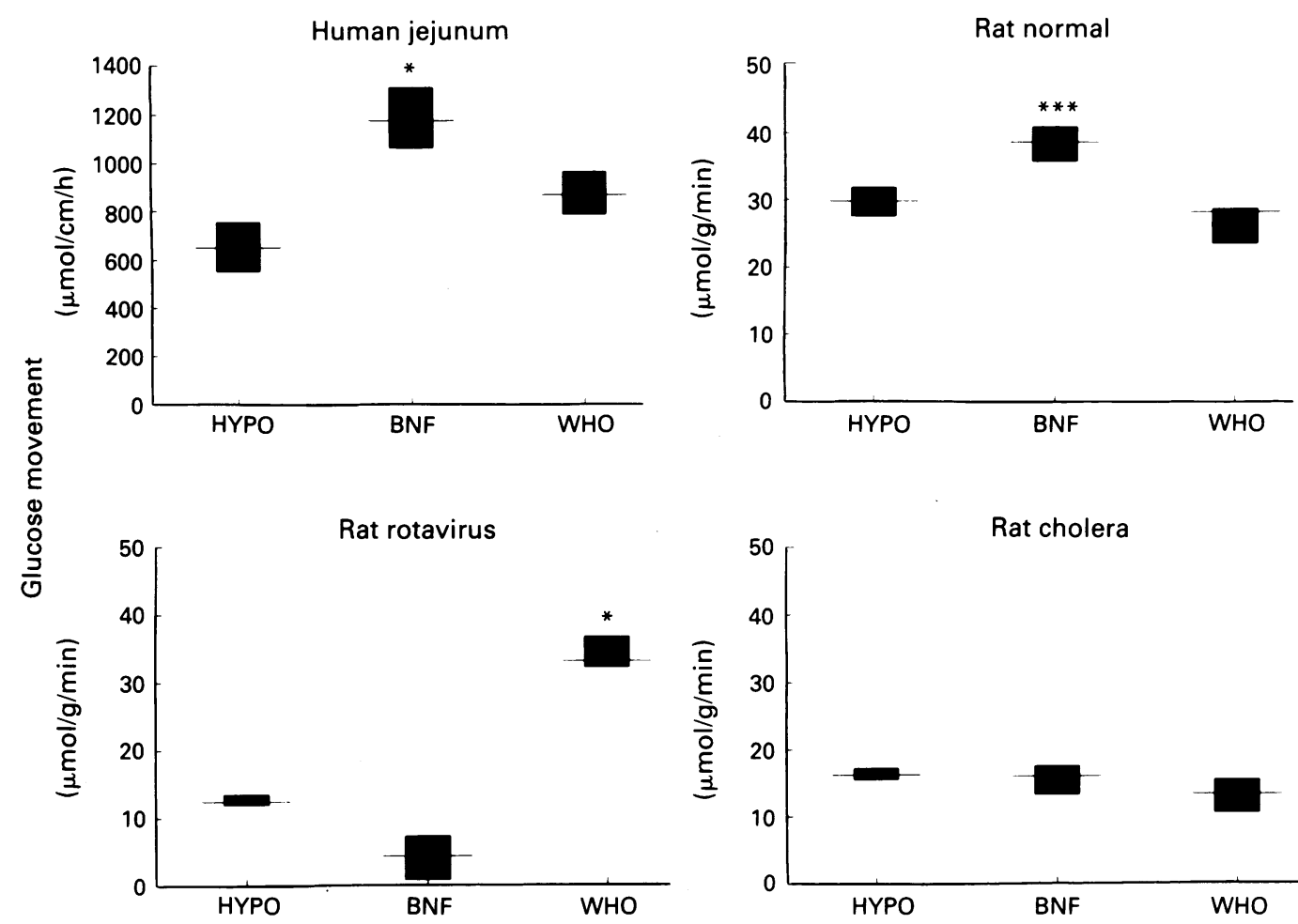

Figure 3. Net glucose absorption during perfusion of hypotonic (HYPO), British National Formulary (BNF) and World Health Organisation (WHO) oral rehydration solutions in four perfusion models (normal human jejunum, normal and cholera toxin treated rat small intestine and rotavirus infected neonatal rat small intestine. ${ }^{\star} p<0 \cdot 001 ;{ }^{\star \star \star} p<0 \cdot 05$.

animal perfusion systems and our own in human jejunum. ${ }^{14} 16$ While net sodium absorption was not different when WHO oral rehydration solution and British National Formulary-oral rehydration solution were perfused in the two non-secreting systems, a difference was apparent in the secreting systems. WHO oral rehydration solution promoted net sodium absorption in the rat rotavirus model but did not reverse net secretion in the rat cholera model. Hypotonicoral rehydration solution induced net water absorption in both secreting models but did not promote net sodium absorption in either. The separation of positive water balance and negative sodium balances in animal perfusion studies have been reported previously. ${ }^{14}$ The ability of hypotonic-oral rehydration solution to stimulate net water absorption without net sodium absorption in the different models is probably the result of passive water absorption, induced by the osmotic gradient produced with this solution. In normal small intestine the reflection coefficient for sodium chloride is between $0 \cdot 4-0 \cdot 9 .{ }^{20}$ If the value is similar in secreting intestine a solution approximating to half normal saline would be absorbed passively, without any contribution from glucose stimulated active transport and thus 'free' water absorption would occur.

The mechanisms by which secretion occurs in the cholera toxin and rotavirus models are different which explains at least in part the differences in absorption seen with WHO-oral rehydration solution in the two models. Cholera toxin inhibits sodium/hydrogen exchange ${ }^{34}$ and stimulates active chloride secretion ${ }^{35}$ which in turn causes passive sodium secretion. In rotavirus infection there is villus shortening and consequent loss of absorptive area but no direct alteration in transport processes. ${ }^{31}{ }^{36}$ In rotavirus infection the villus changes are focal, migrating distally during infection. ${ }^{36}$ Consequently there are areas of intact small intestine in which absorption is able to occur normally. In $V$ cholerae infection the major transport effects are seen in the proximal jejunum. ${ }^{37}$ In our model, however, the whole of the small intestine was exposed to cholera toxin and consequently there was probably extensive inhibition of sodium/ hydrogen counter transport, the major factor in sodium absorption in the fasted state. ${ }^{38}$ Thus, there is greater disruption of the normal active transport system in the cholera toxin model than the rotavirus model. In the former, active sodium absorption from the oral rehydration solutions is solely dependent upon glucose/sodium cotransport, whereas in the rotavirus model both sodium/hydrogen exchange and sodium/glucose cotransport still operate even in shortened villi. The saline perfusion studies showed greater net water and sodium secretion rates in the cholera toxin compared with the rotavirus model which is analogous to the human disease states. In man, cholera results in a stool sodium concentration of $88-140 \mathrm{mmol} / \mathrm{l}$, whereas in rotavirus infection the stool sodium is usually $35 \mathrm{mmol} / \mathrm{l} .^{39}$ Thus, there is both greater sodium secretion and less active sodium absorption in the cholera toxin model than the rotavirus model. These factors combined account for the net negative sodium balance in the former and positive sodium balance in the latter.

Caution must be used in determining the clinical significance of these results. Although sodium secretion was seen with British National Formulary-oral rehydration solution in all perfusion systems, this oral rehydration solution has 
been used widely and a decrease in childhood mortality from acute diarrhoea occurred in England and Wales at a time when its use increased. ${ }^{40}$ The corollary of this is that net positive sodium balance in small intestinal perfusion studies is not necessary for the efficacy of an oral rehydration solution. Indeed a marked positive balance in these models may be hazardous when the solution is used in clinical practice, as such solutions may promote hypernatraemia as occasionally occurs with WHO-oral rehydration solution..$^{40}$ The ability of even low sodium oral rehydration solutions to repair sodium deficits in acute diarrhoea probably results from effective sodium salvage by the terminal ileum and colon. ${ }^{38}$ The contribution of the colon was not studied in any of our models and the ileum was not studied in the human model. Oral rehydration solutions containing 60 $\mathrm{mmol} / \mathrm{l}$ sodium, like hypotonic-oral rehydration solution have, however, been shown to be as effective clinically as higher sodium oral rehydration solutions and are rarely associated with disturbances of plasma sodium concentration. ${ }^{28}$

The goal for the development of future oral rehydration solutions is to produce solutions which are as effective or more so than the existing formulations for correcting dehydration, and which at the same time decrease stool volume and thus water loss. This may be possible, by taking advantage of the high permeability of the proximal jejunum and by using solutions of low osmolality, to maximise passive water absorption. At the same time it is necessary to ensure the delivery of adequate quantities of solute to distal small bowel. While in health glucose does not stimulate a net increase in sodium absorption in the ileum, ${ }^{41}$ the presence of both electrogenic sodium and water absorption has been demonstrated in vitro in human ileum after exposure to cholera toxin ${ }^{42}$ and the same positive effects on sodium balance has been shown with the perfusion of glucose-saline solutions in secreting dog ileum in vivo. ${ }^{43} \mathrm{~A}$ preliminary clinical study with hypotonic-oral rehydration solution indicates that it is effective for rehydration and reduces stool volumes compared with an established slightly hypertonic oral rehydration solution. ${ }^{44}$ This observation supports the view that improved efficacy can be achieved by reducing osmolality of standard glucose electrolyte oral rehydration solutions and gives further credence to the value of animal models for screening new oral rehydration solution formulations.

MJGF gratefully acknowledges support by the Wellcome Trust The authors are grateful to Rorer Health Care Ltd and Nutricia Cow \& Gate for financial support for these studies.

1 Hirschhorn N, Kinzie JL, Sachar DB, Northrup RS, Taylor JO, Ahmed ZS, et al. Decrease in net stool output in cholera during intestinal perfusions with glucose containing solutions. N Englf Med 1968; 279: 176-81.

2 Pierce NF, Banwell JG, Mitra RC, Caravasos GJ, Keimowitz RJ, Mondal A, et al. Effect of intragastric glucose-electrolyte perfusion upon water and electrolyte balance in asiatic cholera. Gastroenterology 1968; 55: 333-43.

3 Pierce NF, Sack BR, Mitra RC, Banwell JG, Brigham JG, Brigham KL, et al. Replacement of water and electrolyte losses in cholera by oral glucose-electrolyte. Ann Intern Med 1969; 70: 1173-81

4 Hirschhorn $\mathrm{N}$. The treatment of acute diarrhoea in children. An historical and physiological perspective. Am $\mathcal{y}$ Clin Nutr 1980; 33: 637-63.

5 Sack DA, Chouwdray AMAK, Eusof A, Merson MH, Islam S, Black RE, et al. Oral rehydration of rotavirus diarrhoea: A double-blind comparison of sucrose and glucose-electrolyte solution. Lancet 1978; ii: $280-3$.

6 Bhargava SK, Sachdev HPS, Das Gupta B, Daral TS, Sing HP, Mohan M. Oral rehydration of neonates and young infants with dehydrating diarrhoea: Comparison of low and standard sodium content in oral rehydration solutions. standard sodium content in oral rehydratr Gastroenterol Nutr 1984; 3: 500-5.

7 Molla AM, Sarker SA, Hossain M, Molla A, Greenough WB Rice powder electrolyte solutions in oral therapy in diarrhoe due to Vibrio cholerae and Escherichia coli. Lancet 1982; 1317-9.

8 Patra FC, Mahalanabis D, Jalan KN, Sen A, Banerjee P. Is oral rice electrolyte solutions superior to glucose electrolyte solution in infantile diarrhoea? Arch Dis Child 1982; 57: 910-2.

9 Kenya PR, Odonga HW, Oundo G, Waswa K, Muttunga J, Molla AM, et al. Cereal based oral rehydration solutions. Arch Dis Child 1989; 64: 1032-5.

10 Alam AN, Sarker SA, Molla AM, Rahaman MM, Greenough WB. Hydrolised wheat based oral rehydration solution for WB. Hydrolised wheat based oral rehydration

11 Aperia AN, Larin L, Zetterstöm R, et al. Salt and wate homeostasis during oral rehydration therapy. $\mathcal{F}$ Pediatr 1983 03: 364-9.

12 Islam MR, Samadi AR, Ahmed SM, Bardham PK, Ali A. Ora rehydration therapy: Efficacy of sodium citrate equals sodium bicarbonate for correction of acidosis in diarrhoea. Gut 1984; 25: 900-4.

13 Santosham M, Burns BA, Reid R, et al. Glycine-based ora rehydration solution: Reassessment of safety and efficacy. F Pediatr 1986; 109: 795-801.

14 Lifshitz F, Wapnir RA. Oral rehydration solutions: experimental optimization of water and sodium absorption. I Pediatr 1985; 106: 383-9.

15 Rolston DDK, Borodo MM, Kelly MJ, Dawson AM, Farthing MJG. Efficacy of oral rehydration solutions in a rat model of secretory diarrhea. 7 Pediatr Gastroenterol Nutr 1987; 6 : 624-30.

16 Hunt JB, Elliott EJ, Farthing MJG. Efficacy of a standard United Kingdom oral rehydration solution (ORS) and a hypotonic ORS assessed by human intestinal perfusion. Aliment Pharmacol Therap 1989; 3: 565-71.

17 Wanitscke R, Georg HJ, Jeschek B, Krog S. The effect of interchange of bicarbonate by citrate in the WHOrecommended oral rehydration solution on net water and solute movement in the human jejunum. Gastroenterolog 1984; 86: 1293.

18 Spiller RC, Jones BJM, Silk DBA. Jejunal water and electrolyte absorption from two proprietary enteral feeds in man: importance of sodium content. Gut 1987; 28: 681-7.

19 World Health Organisation. The treatment and prevention of acute diarrhoea. Practical guidelines. 2nd ed. Geneva: WHO 1989. 46

20 Fordtran JS, Rector FC, Carter NW. The mechanism of sodium absorption in the human small intestine. $\mathcal{f}$ Clin Invest 1968; 47: 884-900.

21 Meeuwisse GW. High sugar worse than high sodium in ora rehydration solutions. Acta Paediatr Scand 1983; 72: 161-6.

22 Sladen GE, Dawson AM. Interrelationship between the absorption of glucose, sodium and water by the norma human jejunum. Clin Sci 1969; 36: 119-32.

23 Modigliani R, Bernier JJ. Absorption of glucose sodium and water by the human jejunum studied by intestinal perfusion with a proximal occluding balloon and at variable flow rates. Gut 1971; 12: 184-93.

24 Nalin DR, Cash RA, Islam R, Molla M, Phillips RA. Ora maintenance therapy for cholera in adults. Lancet 1968; ii 370-2.

25 Elliott EJ, Cunha Ferreira RM, Cameron D, Farthing MJG, Walker-Smith JA. Evaluation of three oral rehydration solutions designed for use in developed communities. Aliment Pharmacol Therap 1989; 3: 233-43.

26 Fordtran JS, Rector FC, Ewton MS, Soter N, Kinney J Permeability characteristics of the human small intestine. f Clin Invest 1965; 44: 1935-44.

27 Raghu BM, Deshpande AP, Chintu C. Oral rehydration for diarrhoeal diseases in children. Trans $R$ Soc Trop Med 1981; 75: 552-5.

28 Elliott EJ, Cunha Ferreira R, Walker-Smith JA, Farthing MJG. Sodium content of oral rehydration solutions: a reappraisal. Gut 1989; 30: 1610-2.

29 Rolston DDK, Moriarty KJ, Farthing MJG, Kelly MJ, Clark ML, Dawson AM. Acetate and citrate stimulate water and solute absorption in the human jejunum. Digestion 1986; 34 : $101-4$.

30 Hoffman SL, Moechtar MA, Scinanjukat CH, Panjabi NH Kunala S, Sutoto, et al. Rehydration and maintenance therapy of cholera patients in Jakata: citrate-based versus bicarbonate-based oral rehydration salt solution. F Infect $D$ is 1985; 152: 1159-65.

31 Salim AFM, Walker-Smith JA, Farthing MJG. Intestinal water and solute transport in a neonatal rat model of rotavirus diarrhoea. Pediatr Res 1989; 26: 280

32 Barclay GR, Turnberg LA. Effect of cold induced pain on salt and water transport in the human jejunum. Gastroenterolog 1988; 94: 994-8.

33 Siegel S, Caslethan NJ. Non parametric statistics for the behavioural sciences. McGraw-Hill, 1988: 206-16.

34 Field M, Fromm D, Al-Awqati Q, Greenough WB. Effects of cholera enterotoxin on ion transport across isolated ileal mucosa. $\mathcal{F}$ Clin Invest 1972; 51: 796-801.

35 mucos. Fin Biochem Soc Trans 1984; 12: 177-80.

36 Graham DY, Sackman JW, Estes MK. Pathogenesis of 
rotavirus induced diarrhoea. Preliminary studies in minature swine piglet. Dig Dis Sci 1984; 29: 1028-35.

37 Banwell TB, Pierce NF, Mitra RC, Brigham KL, Caranasos GJ, Keimowitz RI, et al. Intestinal fluid and electrolyte transport in human cholera. F Clin Invest 1970; 49: 183

38 Powell DW. Intestinal water and electrolyte transport. In: Johnston RL. Physiology of the gastrointestinal tract. New York: Raven Press, 1988: 1267-309.

39 Molla AM, Rahman M, Sarker SA, Molla A. Stool electrolyte content and purging rats in diarrhoea caused by rotavirus, enterotoxigenic $E$.coli and V.cholerae in children. $\mathcal{F}$ Pediatr 1981; 98: 835-8.

40 Walker-Smith JA. Gastroenteritis. In: Walker-Smith JA.
Diseases of the small intestine in childhood. 3rd ed. London: Butterworths, 1988: 185-285.

41 Modigliani R, Berner JJ. Effect du glucose sur les mouvements nets et unidirectionnels de l'eau et des electrolytes dan
l'intestin grele de l'homme. Biol Gastroenterol 1972; 5: 163-

42 Al Awqati Q, Cameron JL, Greenough WB. Electrolyte transport in human ileum: effect of purified choler exotoxin. Am F Physiol 1973; 224: 818-23.

43 Moore WL, Bielwdorf FA, Morawski SG, Finkelstein RA Fordtran JS. Ion transport during cholera toxin induced ileal secretion in the dog. 7 Clin Invest 1971; 50: 312-28.

44 Cleghorn GJ, Shepherd RW, Patrick MK. Comparison of two oral rehydration solutions in children with gastroenteritis in Australia. Clin Therap 1990; 12 (suppl A): 81-5. 